\title{
Propriedades psicométricas da Escala de Responsividade Social-2 para Transtornos do Espectro Autista
}

\author{
Psychometric properties of the Social Responsiveness \\ Scale-2 (SRS-2) for Autism Spectrum Disorder
}

Izabela Guimarães Barbosa', David Henrique Rodrigues,2, Natália Pessoa Rocha', Ana Cristina Simões-e-Silva', Antônio Lúcio Teixeira', Arthur Kummer'

\section{RESUMO}

Objetivo: $O$ objetivo deste trabalho foi avaliar as propriedades psicométricas da versão em português da Escala de Responsividade Social-2 (ERS-2) para crianças e adolescentes com transtorno do espectro autista (TEA). Métodos: A ERS-2 foi respondida pelos pais de 90 pacientes com TEA e 25 controles saudáveis. Análises quanto à validade discriminante, índices de confiabilidade e separação, de adequação e calibração dos itens pelo modelo Rasch foram realizadas. Resultados: $\mathrm{A}$ ERS-2 demonstrou boa consistência interna (alfa de Cronbach $=$ 0,952), um ponto de corte de 41 , sensibilidade de $96,8 \%$, especificidade de $100 \%$ e valor preditivo negativo de 99,9\% para a identificação de TEA. As subescalas apresentaram, de forma geral, adequação ao modelo. No entanto, alguns itens se apresentaram pouco consistentes do ponto de vista estatístico (correlação item-total negativas e misfitting). O mapa de itens mostrou

\section{Palavras-chave}

Transtorno autístico, diagnóstico, psicometria, reprodutibilidade dos testes.

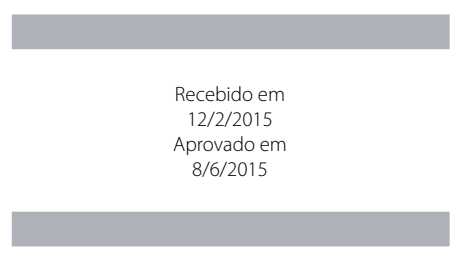

DOI: $10.1590 / 0047-2085000000083$ má cobertura da variável latente, especialmente no espectro mais leve do TEA. Conclusão: Os resultados deste estudo mostraram que a versão em português da ERS-2 pode ser utilizada como ferramenta de triagem para o reconhecimento de TEA em crianças e adolescentes brasileiros. A escala pode ter versões futuras aprimoradas com a substituição dos itens com pior desempenho.

\section{ABSTRACT}

Objective: The purpose of this research was to assess the psychometric properties of the Portuguese version of the Social Responsiveness Scale-2 (SRS-2) for children and adolescents with autism spectrum disorder (ASD). Methods: Parents of 90 patients with ASD and 25 healthy controls responded to the SRS-2. Analyses about the discriminant validity, reliability and separation indexes, fitness and items calibration according to Rasch model were carried on. Results: SRS-2 showed good internal consistency (Cronbach's alpha $=0.952$ ), a cutoff score of 41 , sensitivity of $96.8 \%$, specificity of $100 \%$, and negative predictive value of $99.9 \%$ for the diagnosis of ASD. The subscales generally fitted the model. However, some items presented suboptimal statistics performance (negative item-total correlation and misfitting). The item

1 Universidade Federal de Minas Gerais (UFMG), Faculdade de Medicina, Laboratório Interdisciplinar de Investigação Médica. 2 Universidade Federal de Juiz de Fora (UFJF).

Endereço para correspondência: Izabela G. Barbosa e Arthur Kummer

Laboratório Interdisciplinar de Investigação Médica, Universidade Federal de Minas Gerais, Faculdade de Medicina Av. Prof. Alfredo Balena, 190, 20 andar, sala 281, Santa Efigênia 30130-100 - Belo Horizonte, MG, Brasil E-mail: izabelagb@gmail.com; r2kummer@hotmail.com 


\section{Keywords}

Autistic disorder, diagnosis, psychometrics, reproducibility of results. map showed that the latent variable was not entirely covered by the items, especially on the mildest end of the autistic spectrum. Conclusion: The results of this study showed that the Portuguese version of SRS-2 can be used as a screening tool to improve the recognition of ASD in Brazilian children and adolescents. The scale might have improved versions in the future with the substitution of items with worse performance.

\section{INTRODUÇÃO}

O transtorno do espectro autista (TEA) é um transtorno do neurodesenvolvimento caracterizado por dificuldades sociocomunicativas e problemas específicos do comportamento, como estereotipias, interesses anormais na intensidade ou no foco e alterações de sensibilidade'. O diagnóstico atual de TEA inclui os indivíduos que variam sintomaticamente em um amplo espectro de comprometimento das habilidades sociais e problemas de comportamento, frequentemente se associando a outras alterações do desenvolvimento, como deficiência intelectual e atraso de linguagem.

Estudos epidemiológicos têm mostrado grande aumento na prevalência do TEA, com estimativas recentes de 1 em cada 68 crianças americanas ${ }^{2}$. De forma geral, a prevalência global do TEA aumentou entre 20 e 30 vezes desde os primeiros estudos epidemiológicos², o que tem sido intensamente debatido na literatura. Algumas das possíveis explicações para o aumento da prevalência envolvem um melhor reconhecimento e mudanças nos critérios diagnósticos, incluindo a detecção de casos sem deficiência intelectual ${ }^{3}$. Apesar desse aumento na prevalência, a idade média do primeiro diagnóstico de TEA permanece superior a 4 anos, indicando a necessidade de melhoria na detecção dos quadros, de modo a possibilitar o diagnóstico precoce do TEA².

Para facilitar a detecção precoce do TEA, uma série de instrumentos para triagem desse transtorno foi desenvolvida. Entre os instrumentos utilizados para triar e mensurar a intensidade da sintomatologia do TEA em amostras clínicas e não clínicas, está a Escala de Responsividade Social (ERS), ou Social Responsiveness Scale ${ }^{4,5}$, a qual tem recebido grande atenção. A ERS é um questionário que pode ser respondido entre 15 e 20 minutos, que caracteriza quantitativamente prejuízos de socialização, comunicação, comportamentos repetitivos e interesses restritos que definem o TEA.

Recentemente, uma segunda edição da ERS foi publicada (ERS-2), contendo formulários para diferentes faixas etárias ${ }^{5}$. A ERS coincide com o formulário para crianças e adolescentes (4 a 18 anos) da ERS-2, o qual foi o objeto de estudo deste trabalho. A ERS-2 objetiva triar e quantificar traços do TEA, podendo ser facilmente preenchida por pais e professores. A ERS já foi extensamente traduzida em diversas culturas ${ }^{6-10}$. A ERS tem se mostrado um instrumento válido e confiável ${ }^{6-12}$, mas suas propriedades foram exploradas mais extensamente apenas segundo a Teoria Clássica dos Testes. A ERS não foi suficientemente explorada segundo a Teoria de Resposta ao Item ou de acordo com os modelos Rasch. Na verdade, apenas um estudo realizou uma análise Rasch desse instrumento ${ }^{12}$. Suas propriedades psicométricas ainda não foram analisadas no Brasil. Nesse contexto, o objetivo do presente artigo foi analisar a validade e confiabilidade da ERS-2 na população brasileira, bem como explorar sua estrutura subjacente segundo os modelos Rasch.

\section{MÉTODOS}

\section{Sujeitos}

Trata-se de estudo transversal com um grupo clínico e um grupo controle. O grupo clínico foi composto por uma amostra de conveniência de crianças e adolescentes entre 4 e 18 anos; com diagnóstico confirmado de TEA, de acordo com o DSM-5, de forma independente por dois psiquiatras infantis experientes; atendidos no Ambulatório de Autismo do Serviço de Psiquiatria do Hospital das Clínicas da Universidade Federal de Minas Gerais (UFMG) e que tinham consentido (com seu responsável) em participar da pesquisa. O grupo controle foi composto por crianças e adolescentes entre 4 e 18 anos; recrutados na comunidade local; sem transtornos do desenvolvimento ou problemas comportamentais identificados pelos psiquiatras infantis envolvidos na pesquisa e que tinham consentido (com seu responsável) em participar da pesquisa. Os seus respectivos pais também foram convidados a participar deste estudo e necessitavam ser alfabetizados e compreender o instrumento. Os participantes com dados incompletos foram excluídos do estudo.

Com o objetivo de avaliar a validade discriminante do ERS-2, esse instrumento foi respondido pelos pais de 25 controles e de 31 pacientes com TEA. Em um momento posterior, outros 59 pais de pacientes com TEA também responderam ao ERS-2, cujos dados se somaram aos da amostra anterior de pacientes com TEA e controles, para a análise de confiabilidade e segundo o modelo Rasch.

O protocolo do estudo e os documentos de consentimento informado foram aprovados pelo Comitê de Ética em Pesquisa da UFMG (CAAE: 03655112.3.0000.5149), e todos responsáveis legais pelas crianças deram seu consentimento informado por escrito antes da entrada no estudo.

\section{Tradução e adaptação transcultural da ERS-2}

A versão original em Inglês foi traduzida para o português por um dos pesquisadores (AK). Essa versão foi cotejada com outra versão em português da ERS-2, que estava em posse da editora do instrumento. Uma versão de consenso foi elaborada e retrotraduzida para o inglês por outros dois pesquisado- 
res fluentes em língua inglesa ( $D H R, A L T)$, que não estavam familiarizados com a ERS. Essa versão foi comparada com o original por um leitor nativo inglês, que concluiu que as versões eram idênticas. Nenhuma adaptação cultural foi exigida.

\section{A avaliação clínica dos sintomas relacionados ao comportamento autista}

Os responsáveis legais de todos os participantes responderam à ERS-25. O instrumento foi respondido pelos pais durante a consulta psiquiátrica de rotina de seus filhos com TEA. Os pais dos controles responderam ao instrumento na escola de seus filhos, após convite pela diretora para participarem da pesquisa. Não houve recusa para participação por parte dos pais tanto no grupo com TEA quanto no grupo controle. O instrumento foi entregue aos pais, que responderam de acordo com as informações fornecidas pelo próprio instrumento e podiam solicitar ajuda caso houvesse alguma dúvida durante o procedimento.

A ERS-2 é uma escala de 65 itens que pode ser aplicada aos pais ou aos professores, mensurando o comprometimento sociocomunicativo e comportamental de crianças e adolescentes. Cada item refere-se a um aspecto específico de comportamento sociocomunicativo, sendo pontuado em escala de "1" (não é verdade) a "4" (quase sempre é verdade), posteriormente recodificado em 0-3. A pontuação bruta total varia de 0 a 195. Podem ser geradas pontuação total e pontuações de cinco subescalas (consciência social, cognição social, comunicação social, motivação social e interesses restritos e comportamentos repetitivos).

\section{Análise estatística}

Estatística descritiva foi utilizada para apresentar as características sociodemográficas e clínicas da amostra. Associação entre variáveis dicotômicas foi avaliada com o teste do qui-quadrado de Pearson ou o teste exato de Fisher, quando apropriado. Variáveis contínuas foram expressas como média e desvio-padrão. Todas as variáveis foram testadas quanto à normalidade de distribuição por meio do teste de Shapiro-Wilk, e todos os dados apresentaram distribuição não normal. Portanto, as diferenças entre os dois grupos foram comparadas com o teste de Mann-Whitney.

O escore ótimo da ERS-2 para o diagnóstico de TEA foi calculado por meio da receiver operating characteristic curve (curva ROC). O coeficiente alfa de Cronbach e correlações item-total corrigidas foram computados para verificar a consistência interna da ERS. Todos os valores de $p$ foram bicaudais, e o nível de significância de $a=0,05$ foi escolhido. Essas análises estatísticas foram realizadas com o software SPSS versão 17.0 (SPSS Inc., Chicago, IL, EUA).

Também foram realizadas análises de modelo Rasch utilizando o software Winsteps 3.81.0 (Linacre, Chicago, EUA). Essas análises foram realizadas com as subescalas da ERS-2, considerando que a análise Rasch tem como premissa que é apenas possível gerar medidas verdadeiras de itens que medem o mesmo constructo. Esses itens podem, então, ser ordenados em um contínuo linear com intervalos precisos. O modelo assume que a probabilidade de um respondente endossar um item é uma função logística da diferença entre, por exemplo, a gravidade dos sintomas e a gravidade do sintoma representado pelo item. Desse modo, espera-se que itens que representem sintomas de maior gravidade tenham menor probabilidade de serem endossados pelos casos mais leves. A endossabilidade (tradicionalmente chamada de "dificuldade") de um item é o ponto na escala da variável latente em que as categorias maior e menor têm a mesma probabilidade de serem observadas. No caso, um item considerado "fácil" é mais provável de ser endossado pelos pais de filhos com autismo e os itens mais "difíceis" serão endossados provavelmente apenas pelos casos de maior gravidade.

As análises Rasch geram variáveis de ajuste (fit statistics) que permitem avaliar a adequação dos itens e das pessoas (respondentes) ao modelo. Essas análises são apresentadas sob a forma de quadrados médios (MnSq) de infit e outfit. O valor esperado de infit e outfit é de 1, mas valores entre 0,5 e 1,5 ainda são aceitáveis ${ }^{13}$. Estatísticas de infit e outfit t-padronizados (" $\mathrm{t}$ ") também são de interesse, com valores esperados próximos a zero e valores absolutos aceitáveis de até $3^{13}$. Coeficientes de separação e confiabilidade das pessoas e dos itens também foram calculados pela análise Rasch. A confiabilidade das pessoas pela teoria Rasch é similar à "confiabilidade do teste" (por exemplo: alfa de Cronbach) pela teoria clássica do teste. Porém, de modo geral, o alfa de Cronbach superestima a confiabilidade, enquanto a confiabilidade Rasch a subestima. A confiabilidade dos itens não possui equivalente pela teoria clássica do teste ${ }^{13}$.

O coeficiente de separação de pessoas é um índice da precisão com que a variabilidade presente nos pacientes é capturada pelo teste. Valores de separação de pessoas menores que 2 (geralmente associado a uma confiabilidade $<0,8)$ implicam que o instrumento pode não ser sensível o suficiente para distinguir entre pacientes de maior e menor gravidade, podendo ser necessários mais itens. A separação do item é utilizada para verificar a hierarquia de itens. Separação de itens baixa $(<3$, com confiabilidade de itens $<0,9)$ implica que a amostra pessoa não é grande o suficiente para confirmar a hierarquia de dificuldade de item do instrumento. Os índices de separação são medidas interessantes de confiabilidade quando as próprias medidas de confiabilidade se aproximam de 1, exercendo efeito teto.

\section{RESULTADOS}

\section{Dados sociodemográficos e clínicos da população estudada}

Não houve diferença estatisticamente significativa em relação a idade, gênero e idade materna entre pacientes e controles 
(Tabela 1). A média de idade dos pacientes foi de 9,40 \pm 4,87 anos e a do grupo controle foi de 9,24 $\pm 3,47$ anos. A maioria dos sujeitos incluídos neste estudo foi do sexo masculino.

Conforme esperado, os pacientes com TEA apresentaram pontuações significativamente mais elevadas na ERS-2 quando comparados aos controles saudáveis (103,82 $\pm 26,3$ versus $26,40 \pm 10,8 ; p<0,001)$. Pacientes com TEA apresentaram pontuações significativamente mais elevadas em todas as subescalas $(p<0,001)$.

Tabela 1. Dados sociodemográficos e clínicos da população de pacientes do TEA em comparação com controles

\begin{tabular}{|c|c|c|c|}
\hline & $\begin{array}{l}\text { Pacientes com TEA } \\
\quad(\mathrm{N}=90)\end{array}$ & Controles $(\mathrm{N}=25)$ & pvalue \\
\hline Idade em anos (média \pm DP) & $9,40( \pm 4,87)$ & $9,24( \pm 3,47)$ & $0,70^{\mathrm{a}}$ \\
\hline $\begin{array}{l}\text { Gênero masculino } \\
\text { (frequência) (\%) }\end{array}$ & 83,87 & 76,0 & $0,27^{\mathrm{b}}$ \\
\hline Idade materna (média \pm DP) & $30,59( \pm 6,17)$ & $27,58( \pm 7,42)$ & $0,08^{\mathrm{a}}$ \\
\hline ERS-2 (média \pm DP) & $103,82( \pm 26,3)$ & $26,40( \pm 10,8)$ & $<0,001^{\mathrm{a}}$ \\
\hline $\begin{array}{l}\text { Consciência social (média } \\
\pm D P \text { ) }\end{array}$ & $12,12( \pm 4,02)$ & $3,40( \pm 2,70)$ & $<0,001^{\mathrm{a}}$ \\
\hline $\begin{array}{l}\text { Cognição social (média } \\
\pm D P \text { ) }\end{array}$ & $19,85( \pm 5,49)$ & $5,30( \pm 3,77)$ & $<0,001^{\mathrm{a}}$ \\
\hline $\begin{array}{l}\text { Comunicação social (média } \\
\pm \text { DP) }\end{array}$ & $34,08( \pm 9,99)$ & $7,25( \pm 3,93)$ & $<0,001^{\mathrm{a}}$ \\
\hline $\begin{array}{l}\text { Motivação social (média } \\
\pm \text { DP) }\end{array}$ & $17,69( \pm 6,39)$ & $9,00( \pm 3,56)$ & $<0,001^{\mathrm{a}}$ \\
\hline Maneirismos (média $\pm \mathrm{DP}$ ) & $20,08( \pm 7,70)$ & $1,45( \pm 1,84)$ & $<0,001^{\mathrm{a}}$ \\
\hline
\end{tabular}

DP: desvio-padrão; ERS-2: Escala de Responsividade Social-2; TEA: transtorno do espectro autista; ‘ teste de MannWhitney; ${ }^{b}$ teste do qui-quadrado; ${ }^{\mathrm{c}}$ teste de Fisher.

\section{Validade discriminante da ERS-2}

A análise da curva ROC da ERS-2 mostrou uma área sob a curva de 0,99 [intervalo de confiança (IC) a 95\% = 0,98-1,00]. Utilizando-se como ponto de corte 41, a ERS-2 apresentou sensibilidade de $96,8 \%$, especificidade de $100 \%$, valor preditivo positivo (VPP) de 100\% e valor preditivo negativo (VPN) de $99,9 \%$ (Figura 1).

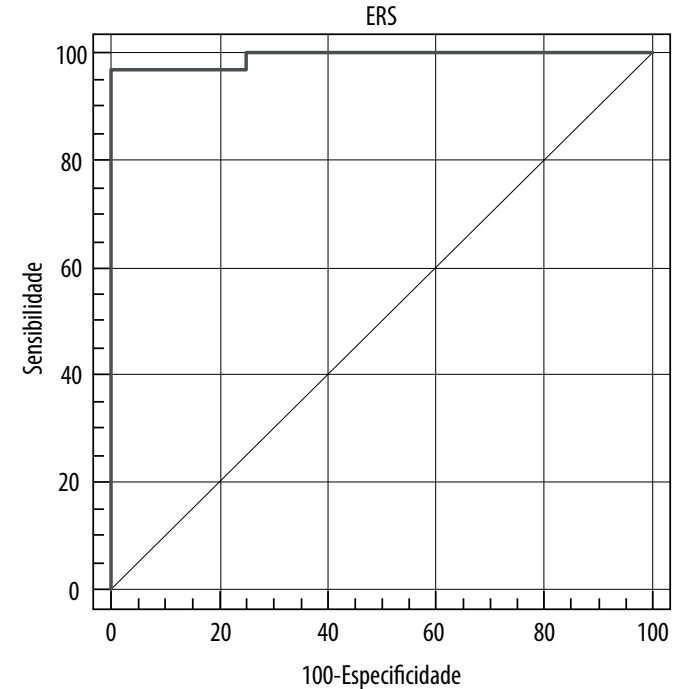

Figura 1. Receiver operating characteristic curve (curva ROC) da Escala de Responsividade Social-2 (ERS-2) para avaliação de transtorno do espectro autista.

\section{Consistência interna da ERS-2}

O alfa de Cronbach para a ERS-2 foi de 0,952. Os alfas para as subescalas variaram de 0,671 a 0,868. O item 41 ("Muda sem objetivo de uma atividade para outra") apresentou pequena correlação negativa com sua subescala de Comunicação social $(-0,020)$ e positiva com a escala total $(0,042)$. O item 43 ("Se separa facilmente dos cuidadores") apresentou pequena correlação negativa com a escala ERS-2 $(-0,022)$ e positiva com a subescala de Motivação social $(0,012)$. No entanto, a exclusão desses dois itens não aumenta significativamente o alfa de Cronbach da escala $(0,954)$.

\section{Análises Rasch}

As subescalas da ERS-2 apresentaram valores médios de MnSq de infit e de outfit, bem como de infit e outfit t-padronizados (t), adequados para as pessoas e para os itens (Tabela 2). Analisados individualmente, alguns itens apresentam misfit, ou seja,

Tabela 2. Valores de MnSq de infit e de outfit, de infit e outfit t-padronizados (t), de separação e de confiabilidade das subescalas da Escala de Responsividade Social-2 (ERS-2) para pessoas e itens

\begin{tabular}{|c|c|c|c|c|c|c|c|}
\hline Subescala & & MnSq infit & tinfit & MnSq outfit & toutfit & Separação & Confiabilidade \\
\hline \multirow[t]{2}{*}{ Consciência social } & Pessoas & 1,04 & $-0,1$ & 1,05 & $-0,1$ & 1,32 & 0,63 \\
\hline & Itens & 1,01 & 0,0 & 1,05 & 0,2 & 3,78 & 0,93 \\
\hline \multirow[t]{2}{*}{ Cognição social } & Pessoas & 1,07 & $-0,1$ & 1,12 & 0,0 & 1,48 & 0,69 \\
\hline & Itens & 0,99 & $-0,2$ & 1,12 & 0,3 & 3,68 & 0,93 \\
\hline \multirow[t]{2}{*}{ Comunicação social } & Pessoas & 1,11 & $-0,1$ & 1,14 & 0,0 & 2,21 & 0,83 \\
\hline & Itens & 1,02 & $-0,1$ & 1,14 & 0,5 & 3,93 & 0,94 \\
\hline \multirow[t]{2}{*}{ Motivação social } & Pessoas & 1,02 & 0,0 & 1,05 & 0,0 & 1,63 & 0,73 \\
\hline & Itens & 1,02 & 0,0 & 1,05 & 0,0 & 4,22 & 0,95 \\
\hline \multirow{2}{*}{$\begin{array}{l}\text { Interesses restritos } \\
\text { e comportamentos } \\
\text { repetitivos }\end{array}$} & Pessoas & 1,04 & $-0,1$ & 1,03 & $-0,1$ & 1,92 & 0,79 \\
\hline & Itens & 1,00 & 0,0 & 1,03 & 0,2 & 2,10 & 0,81 \\
\hline
\end{tabular}


valores de MnSq de infit e de outfit inadequados. Nas subescalas de Consciência social, Cognição social e Motivação social, um item apresenta misfit (itens 56, 59 e 43, respectivamente). Já na escala de Comunicação social, cinco itens apresentam misfit (41, 60, 57, 46 e 55, em ordem decrescente de misfitting).

De forma geral, os itens apresentaram boa ordenação dos limiares de categorias de resposta aos itens e estatísticas de adequação (fit statistics) (Tabela 3).

A análise dos mapas dos itens das subescalas, que associam a gravidade dos sintomas (endossabilidade ou "dificuldade" dos itens) com a gravidade dos pacientes (ou "habilidade"), revelou que os itens mais fáceis não eram endossados por participantes do grupo controle. Além disso, os mapas das subescalas também mostraram que havia pacientes de gravidade maior que a apontada pelos itens da ERS-2, ou seja, há um efeito teto da escala que dificulta maior discriminação dos casos mais graves quanto à gravidade dos domínios sintomatológicos do autismo. O mapa de itens da escala completa apresentou melhor cobertura dos pacientes mais graves, mas ainda possui poucos itens capazes de avaliar manifestações mais sutis de dificuldades sociocomunicativas na população geral. Por limitações de espaço, apenas o mapa de itens da escala completa foi exibido (Figura 2), ilustrando o direcionamento pobre da "dificuldade" dos itens à "habilidade" dos respondentes da ERS-2.

Tabela 3. Ordenação dos limiares de categorias de resposta aos itens e estatísticas de adequação das categorias

\begin{tabular}{lccccc}
\hline Subescala & Categorias & Limiar & Erro-padrão & Infit & 0utfit \\
\hline Consciência social & 0 & & & & \\
& 1 & $-0,13$ & 0,09 & 0,85 & 0,77 \\
& 2 & 0,05 & 0,08 & 0,79 & 0,66 \\
Cognição social & 3 & 0,08 & 0,09 & 1,10 & 1,32 \\
& 0 & & & & \\
& 1 & 0,37 & 0,07 & 0,91 & 0,79 \\
Comunicação & 2 & $-0,11$ & 0,07 & 0,74 & 0,60 \\
social & 3 & $-0,26$ & 0,07 & 1,10 & 1,49 \\
& 1 & & & & \\
& 2 & 0,11 & 0,05 & 0,85 & 0,62 \\
Motivação social & 0 & 0,02 & 0,05 & 0,87 & 0,71 \\
& 1 & $-0,01$ & 0,07 & 1,05 & 1,22 \\
& 2 & $-0,03$ & 0,07 & 0,85 & 0,70 \\
rempetitivos & 2 & 0,10 & 0,07 & 0,74 & 0,62 \\
& 3 & 0,12 & 0,07 & 1,04 & 1,19 \\
\hline Interesses & 3 & 0,05 & 0,08 & 1,06 & 1,05 \\
& 0 & & & & \\
& 1 & $-0,21$ & 0,08 & 0,91 & 0,91 \\
& 2 & & & &
\end{tabular}

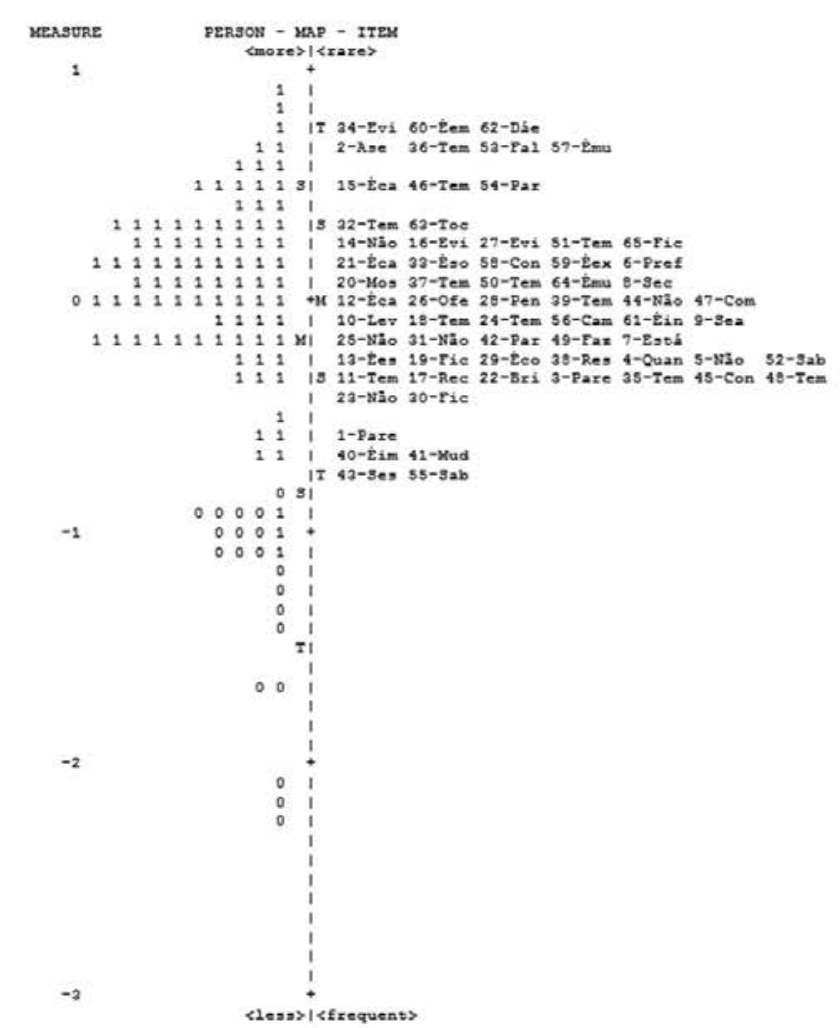

0: grupo controle; 1: autismo.

Figura 2. Mapa de itens da Escala de Responsividade Social-2 (ERS-2).

\section{DISCUSSÃO}

A amostra americana clínica de TEA e de irmãos não afetados possui médias do escore bruto da ERS-2 (106,6 e 24,6, respectivamente) bastante similares à deste estudo (103,82 e 26,40, respectivamente). A versão em português da ERS-2 mostrou ponto de corte $(\geq 41)$ abaixo do relatado pelos autores originais da referida escala ${ }^{2}$. A interpretação da versão em inglês da ERS-2 é dada a partir do escore T da escala, ou seja, o escore bruto é convertido para um escore normatizado para aquela população. O ponto de corte para a identificação do autismo por meio da ERS-2 para a faixa etária de 4 a 18 anos é o escore $\geq 60 T$. Esse escore T é equivalente ao escore bruto de 58-60 na escala para pais de meninos, e ao escore de 5253 na escala para pais de meninas. 0 equivalente do escore bruto de 41 na escala T americana é 55T para meninos e 57T para meninas. No estudo americano, o escore bruto de 60, para ambos os gêneros, fornece uma sensibilidade de $93 \%$ e especificidade de $91 \%$, valores ligeiramente inferiores aos encontrados neste trabalho. Outros trabalhos encontraram pontos de corte intermediários a esses, mantendo boas propriedades discriminativas. Roeyers et al., com uma amostra de crianças belgas e holandesas ${ }^{10}$, encontraram sensibilidade de $90 \%$ e especificidade de $88 \%$ com o ponto de corte de 51 . Kamio et al., testando a versão japonesa da ERS ${ }^{6}$, encontra- 
ram sensibilidade de 91\%/89\% e especificidade de 48\%/41\% com os pontos de corte de 53,5/52,5 para meninos/meninas, correspondendo a um escore $T$ de 60T/62T.

A ERS-2 também apresentou excelente consistência interna de acordo com o alfa de Cronbach, similar à versão original $(0,95)^{5}$. Wigham et al. e Duku et al. relatam um alfa simi$\operatorname{lar}(0,92 \text { e 0,93, respectivamente) })^{11,12}$. No estudo de Wigham et al., o alfa das subescalas variou de 0,47 a 0,83, enquanto no de Duku et al. o alfa variou de 0,60 a 0,85. Em ambos os trabalhos, as subescalas com menor alfa (consciência social) e maior alfa (comunicação social) foram as mesmas deste trabalho, que são as subescalas com menor e maior número de itens, respectivamente ${ }^{11,12}$.

Um dos pontos relevantes da análise Rasch apresentada neste trabalho é ressaltar que uma escala de avaliação clínica do autismo possui itens de graus de endossabilidade ("dificuldade" ou gravidade do sintoma) diferentes. Ou seja, contrariamente ao postulado pela avaliação tradicional, itens que indicam maior gravidade não deveriam ter o mesmo peso no escore total da escala do que itens sobre sintomas de menor gravidade.

Além disso, os mapas de itens sugerem que as subescalas poderiam ser aprimoradas com a inclusão de itens mais "fáceis", que também possam ser endossados por crianças com desenvolvimento típico, e itens mais "difíceis", que permitam melhor diferenciação entre os casos mais graves, de modo que o "espectro autista" possa ser avaliado de forma mais adequada. De fato, as subescalas, com exceção da escala de comunicação social, apresentaram coeficientes de separação de pessoas baixos, reforçando que as subescalas da ERS-2 podem ser pouco sensíveis para distinguir diferentes níveis de gravidade.

No entanto, a inclusão desses itens poderia aumentar substancialmente o tamanho do instrumento, tornando-o inadequado como instrumento de triagem. Apesar disso, a escala completa apresenta razoável cobertura da habilidade dos pacientes autistas, o que também pode ser conferido pela média da distribuição de itens e pessoas (marcada como M na figura 2), mostrando-se pouco acurada apenas na avaliação de manifestações sutis de dificuldades sociocomunicativas da população sem autismo. Uma possível solução para a melhoria da escala seria a substituição de alguns itens que se acumulam em certas faixas da variável latente por outros itens que consigam avaliar as faixas descobertas do espectro autista.

Alguns procedimentos da análise clássica dos itens e da análise Rasch podem ser combinados na avaliação da qualidade dos itens que compõem uma escala. Linacre sugere que inicialmente se identifiquem itens com correlações item-total negativas ${ }^{13}$. Nesse sentido, os itens 41 ("Muda sem objetivo de uma atividade para outra") e 43 ("Se separa facilmente dos cuidadores") são passíveis de revisão. Além disso, uma análise da validade de conteúdo desses itens, de fato, gera questionamentos sobre sua real contribuição à identificação de dificuldades sociocomunicativas.

Um segundo passo sugerido por Linacre se refere aos valores de infit e outfit. Nesse sentido, valores aberrantes de outfit são mais importantes do que de infit; MnSq são mais relevantes que os valores de t; e underfit (valores muito acima de 1) é pior do que overfit (valores muito abaixo de 1) ${ }^{13}$. A análise de misfitting dos itens permite identificar alguns itens também passíveis de revisão. Os mesmos itens que apresentaram correlação item-total negativa lideram a lista de misfitting. Além de misfitting, alguns itens apresentam validade de conteúdo também questionável, como o item 59 ("É muito desconfiado") e 46 ("Tem expressões faciais muito sérias"). Poucos trabalhos realizaram análise similar da ERS-2. No estudo de Duku et al., um total de 35 itens chegou a ser excluído do instrumento, por razões que variam de misfitting a limiares desordenados ${ }^{12}$. Nesse trabalho, os autores haviam realizado uma análise Rasch de acordo com o modelo de crédito parcial, que analisa a ordenação dos itens individualmente. Na presente pesquisa, a análise foi realizada segundo o modelo de escala de avaliação (rating scale), que se adequa melhor à ERS-2. No entanto, essa abordagem faz uma análise geral da ordenação dos itens da escala ou subescala, a qual não apresentou desordem. De todo modo, os 30 itens restantes do estudo de Duku et al. apresentaram propriedades psicométricas melhores do que os 65 itens $^{12}$. Infelizmente os autores não realizaram a análise pelo mapa de itens para saber se esses itens eram capazes de avaliar adequadamente a variável latente conforme se apresenta na população.

Apesar dessas limitações da ERS-2, este trabalho confirma suas propriedades discriminativas para a identificação do TEA. Há poucos estudos sobre a prevalência do TEA no Brasil e sua frequência é possivelmente subestimada. O único estudo que investigou a prevalência de TEA no Brasil estimou uma taxa de $0,3 \%{ }^{14}$. A identificação precoce dos TEA é importante para estimular as habilidades sociais, de comunicação e outras habilidades cognitivas, além de reduzir comportamentos repetitivos e estereotipados ${ }^{15}$.

A ERS-2 é um instrumento que pode ser utilizado por pais e professores, sendo ainda necessária sua validação no Brasil com seu preenchimento pelos profissionais de educação. A identificação precoce está associada à criação de ambiente em que todas as pessoas que interagem de forma regular com o indivíduo com TEA tenham maior conhecimento a respeito das alterações e dos comportamentos associados ao TEA. Dessa forma, a identificação precoce é um dos principais preditores de resultado funcional e de adaptação social de pacientes com TEA ${ }^{15}$. Ressalta-se que a aplicação do instrumento em professores mostrou propriedades psicométricas semelhantes àquelas obtidas pelo relato dos pais?.

Há limitações neste estudo que devem ser mencionadas. Este estudo incluiu uma amostra restrita de pacientes com TEA, dificultando a normatização de seus escores conforme 
proposto pelo manual do instrumento. Entretanto, isso não impediu o uso de escores brutos para a triagem do TEA. Os pacientes também foram recrutados em um centro terciário, onde os pacientes mais complexos e com sintomas mais pronunciados do TEA são normalmente atendidos. Considerando que a ERS-2 afere quantitativamente sintomas autísticos, pontuações menores que 41 não necessariamente significam ausência de sintomas do TEA, podendo associarse a algumas dificuldades sociocomunicativas e de comportamento ${ }^{16}$. Além disso, os pontos de corte podem ser diferentes caso o grupo com TEA seja dividido de acordo com os especificadores de nível de desenvolvimento intelectual e de linguagem, o que merece investigação em estudos futuros. De fato, suspeita-se de que esses especificadores e problemas de comportamento influenciem a pontuação da ERS, pois alguns itens da escala podem avaliar sintomas que não são específicos do TEA ${ }^{17}$. Nesse sentido, a escala poderia avaliar não somente a gravidade dos traços autísticos de uma criança, mas também a gravidade de problemas associados $^{18}$. Por fim, o formulário da ERS-2 utilizado avalia uma faixa etária ampla (4-18 anos) em que, mesmo em pessoas com desenvolvimento típico, há grande variação nas habilidades sociocomunicativas, de linguagem e cognitivas de forma geral. No TEA, essa variabilidade é ainda maior. Desse modo, não se tem clareza sobre como informantes respondem (ou deveriam responder) a itens que não se aplicam a suas crianças (por exemplo: itens avaliando conversação em uma criança não verbal) ${ }^{18}$. Por outro lado, apesar dessas limitações, a amostragem consecutiva, características da amostra similares à do TEA na população geral (por exemplo: maior proporção do gênero masculino) e a avaliação de pacientes por clínicos experientes são os pontos fortes do estudo.

\section{CONCLUSÃO}

A ERS-2 é um instrumento simples e de rápida aplicação, que pode ser útil na pesquisa e prática clínica. Os resultados do presente estudo corroboram a validade discriminante da ERS-2, com um ponto de corte 41 fornecendo ótimos índices de sensibilidade e especificidade. A ERS-2 também apresenta boa consistência interna, mas alguns itens apresentam correlação item-total e valores de infit e de outfit inadequados. Além disso, a escala não possui itens capazes de avaliar adequadamente manifestações mais sutis de dificuldade sociocomunicativa. Desse modo, alguns itens da escala que apresentam elevado misfit poderiam ser substituídos por outros itens que cobrissem mais adequadamente o extremo da escala que avalia quadros leves de TEA. Estudos futuros devem buscar normatizar, aprimorar e reavaliar as propriedades psicométricas da ERS-2, tentando envolver pais, professores e indivíduos com TEA de diferentes níveis de funcionamento intelectual e de linguagem.

\section{CONTRIBUIÇÕES INDIVIDUAIS}

Izabela Guimarães Barbosa - Foi responsável pela coleta de dados clínicos, redação do artigo e aprovou a versão final.

David Henrique Rodrigues - Foi responsável pela coleta de dados clínicos, da tradução da escala e aprovou a versão final.

Natália Pessoa Rocha - Participou da coleta de dados clínicos e aprovou a versão final.

Ana Cristina Simões-e-Silva - Participou da elaboração do projeto, coordenou a redação do artigo e aprovou a versão final.

Antônio Lúcio Teixeira - Supervisor acadêmico responsável pela elaboração do trabalho dos autores principais, participou da elaboração do projeto, da tradução da escala e aprovou a versão final.

Arthur Kummer - Coordenou a coleta de dados clínicos, análise de dados, a tradução da escala e a redação do artigo, e aprovou a versão final.

\section{CONFLITOS DE INTERESSE}

Não há.

\section{AGRADECIMENTOS}

Este trabalho foi financiado pelo Conselho Nacional de Desenvolvimento Científico e Tecnológico (CNPq, Brasil), Fundação de Amparo à Pesquisa do Estado de Minas Gerais (Fapemig, Brasil) e Coordenação de Aperfeiçoamento de Pessoal de Nível Superior (Capes, Brasil). Dra. Izabela Guimarães Barbosa recebeu bolsa de estudos da Capes para a realização de seu pós-doutorado.

\section{REFERÊNCIAS}

1. American Psychiatric Association. Diagnostic and statistical manual of mental disorders (5th ed.). Washington, DC: American Psychiatric Publishing; 2013.

2. Developmental Disabilities Monitoring Network Surveillance Year 2010 Principal Investigators; Centers for Disease Control and Prevention (CDC). Prevalence of autism spectrum disorder among children aged 8 years - autism and developmental disabilities monitoring network, 11 sites, United States, 2010. MMWR Surveill Summ. 2014;63(2):1-21.

3. Levy SE, Mandell DS, Schultz RT. Autism. Lancet. 2009;374(9701):1627-38.

4. Constantino JN, Davis SA, Todd RD, Schindler MK, Gross MM, Brophy SL, et al. Validation of a brief quantitative measure of autistic traits: comparison of the social responsiveness scale with the autism diagnostic interview-revised. J Autism Dev Disord. 2003;33(4):427-33.

5. Constantino JN, Gruber CP. Social Responsiveness Scale - Second Edition (SRS-2). Torrance, CA: Western Psychological Services; 2012.

6. Kamio Y, Inada N, Moriwaki A, Kuroda M, Koyama T, Tsujii H, et al. Quantitative autistic traits ascertained in a national survey of 22529 Japanese schoolchildren. Acta Psychiatr Scand. 2013;128(1):45-53.

7. Fombonne E, Marcin C, Bruno R, Tinoco CM, Marquez CD. Screening for autism in Mexico. Autism Res. 2012;5(3):180-9. 
8. Wang J, Lee LC, Chen YS, Hsu JW. Assessing autistic traits in a Taiwan preschool population: cross-cultural validation of the Social Responsiveness Scale (SRS). J Autism Dev Disord. 2012;42(11):2450-9.

9. Bölte S, Poustka F, Constantino JN. Assessing autistic traits: cross-cultural validation of the social responsiveness scale (SRS). Autism Res. 2008;1(6):354-63.

10. Roeyers H, Thys M, Druart C, De Schryver M, Schittekatte M. SRS screeninglijst voor autismespectrum stoornissen. Amsterdam: Hogrefe; 2011.

11. Wigham S, McConachie H, Tandos J, Le Couteur AS; Gateshead Millennium Study core team. The reliability and validity of the Social Responsiveness Scale in a UK general child population. Res Dev Disabil. 2012;33(3):944-50.

12. Duku E, Vaillancourt T, Szatmari P, Georgiades S, Zwaigenbaum L, Smith IM, et al.; Pathways in ASD Study Team. Investigating the measurement properties of the social responsiveness scale in preschool children with autism spectrum disorders. J Autism Dev Disord. 2013;43(4):860-8.
13. Linacre JM. A user's guide to WINSTEPS and ministep Rasch model computer programs: Program manual 3.81. Chicago: Winsteps; 2014.

14. Paula CS, Ribeiro SH, Fombonne E, Mercadante MT. Brief report: prevalence of pervasive developmental disorder in Brazil: a pilot study. J Autism Dev Disord. 2011;41(12):1738-42.

15. Fernell E, Eriksson MA, Gillberg C. Early diagnosis of autism and impact on prognosis: a narrative review. Clin Epidemiol. 2013;5:33-43.

16. Constantino JN, Todd RD. Intergenerational transmission of subthreshold autistic traits in the general population. Biol Psychiatry. 2005;57(6):655-60

17. Grzadzinski R, Di Martino A, Brady E, Mairena MA, O’Neale M, Petkova E, et al. Examining autistic traits in children with ADHD: does the autism spectrum extend to ADHD? I Autism Dev Disord. 2011;41(9):1178-91.

18. Hus V, Bishop S, Gotham K, Huerta M, Lord C. Factors influencing scores on the social responsiveness scale. J Child Psychol Psychiatry. 2013;54(2):216-24. 